\title{
Impacted Teeth and Mandibular Fracture
}

Murat Metina, DDS, PhD

İsmail Sener ${ }^{b}$, DDS, PhD

Mustafa Tek', DDS, PhD

\section{ABSTRACT}

Objectives: In this retrospective study, we measured the relationship between the presences of impacted or unerupted teeth in the mandible and mandibular fractures.

Methods: The records and radiographs of 41 patients with mandibular fracture associated with impacted or unerupted teeth were examined. The presence of impacted or unerupted teeth were assessed for each patient and related to the occurrence of fractures of mandible.

Results: Patients with fracture in the impacted or unerupted teeth area present had a 1,73 times greater chance of an mandibular fracture than patients with no fracture in the impacted or unerupted teeth area. There was a statistically significant variation in the risk for a mandibular fracture depending on impacted or unerupted teeth presence $\left(\mathrm{x}^{2}=5.29, \mathrm{P}<.05\right)$.

Conclusion: The presence of an impacted or unerupted teeth significantly increases the likelihood of an mandibular fracture. (Eur J Dent 2007;1:18-20)

Key words: Mandibular fracture; Impacted teeth; Unerupted teeth.

\section{INTRODUCTION}

The most common facial bones fracture is the mandible fracture. ${ }^{1}$ Mandibular fracture patterns depend on multiple factors, including direction and amount of force, presence of soft tissue bulk, and biomechanical characteristics of the mandible such as bone density and mass or anatomic structures creating weak areas. ${ }^{2,3}$

The hypothesis that the presence of mandibular third molars (M3) is associated with an increased risk on angle fractures. This hypothesis is supported by biomechanical and epidemiologic studies. In human clinical studies, the presence of the M3 has been repetitively shown to be associated with higher relative risk for angle fracture. These studies demonstrated that whenever the M3

\footnotetext{
$\square$ a Associate Professor, Dental Hospital. Bursa, Turkey

${ }^{b}$ Dr, Dental Hospital. Samsun, Turkey

c Dr, Dental Hospital. Karșıyaka-İzmir, Turkey

$\square$ Corresponding author: Dr. İsmail ȘENER

Aḡız ve Diş Saḡlıḡı Merkezi

Tel: +903624400045

E-Mail: isenerahotmail.com
}

was present, the risk of angle fracture increased 2-to 3-fold when it was compared with absence at M3.4-6 An impacted tooth is one that fails erupt into the dental arch within the expected time. The unerupted term includes both impacted teeth and erupting teeth. ${ }^{7}$

The impacted or unerupted M3 could weaken the mandible because the tooth occupies more osseous space. ${ }^{8}$ However, it is not known if the other impacted or unerupted teeth weaken the mandible or not. There is not any study supports the hypothesis which is pointing out the relationship between the presences of mandibular impacted or unerupted teeth and an increasing risk on mandibular fractures. In this study, we assessed the relationship between impacted or unerupted and mandibular fractures.

\section{MATERIALS AND METHODS}

A retrospective review was conducted of patients who presented with fractured mandibles at University of Ondokuz Mayıs, Department of Oral and Maxillofacial Surgery, between 1995 and 2003. 
One hundred one patients having mandibular fractures and forty-one patients having impacted or unerupted teeth were identified.

To assess the predictor variable, panoramic radiographs were used (presence or absence of impacted or unerupted teeth) and conclusion variable (presence or absence of mandibular fracture). In addition, with the help of the patients' hospital charts and panoramic radiographs, their age, gender, type of fracture, radiologic evaluation, and amount of impacted or unerupted teeth were assessed. The severity of tooth impaction was classified as either "complete" or "partial bony".

In order to show a causal relationship between impacted or unerupted teeth and mandibular fractures, each fracture was divided into two groups. Group I consisted of mandibular fractures which is related to impacted or unerupted teeth. Group II also consisted of mandibular fractures but they are not related to impacted or unerupted teeth.

The database was analyzed with the use of the SPSS version 10.0. To assess the relationships between the presence of impacted or unerupted teeth and the risk of mandibular fractures, the test of comparison of proportions was computed. If $\mathrm{P}<.05$ was observed, differences were considered to be statistically significant.

Table 1. Demographic data by impacted or unerupted teeth presence/absence.

\begin{tabular}{lccc}
\hline Variables & Group 1 & Group 2 & P \\
\hline Patients & 26 & 15 & - \\
Sex & & & \\
Male & 18 & 13 & \\
Female & 8 & 2 & .44 \\
Age & & & \\
$\leq 20$ years & 18 & 8 & \\
$21-30$ years & 4 & 7 & \\
$31-40$ years & 3 & - & \\
$\geq 40$ years & 1 & - & .78 \\
Etiology & & & \\
Falls & 10 & 8 & \\
Fight & 6 & 3 & \\
MVAs & 2 & 1 & \\
Sports & 2 & 1 & \\
Other & 6 & 2 & - \\
Fracture site & & & \\
Angle & 14 & 2 & \\
Body & 9 & 3 & \\
Symphysis & 4 & 5 & \\
Parasymphysis & 5 & 2 & \\
Orher & 3 & 5 & - \\
\hline MVAs:Mor & & & \\
\hline
\end{tabular}

MVAs: Motor Vehicle Accident.

\section{RESULTS}

The study was continued on 41 patients having impacted or unerupted teeth. The patients are at the age of 2 to 45 years (mean age, 17.51 years). 31 male and 10 female samples were acquired. The 41 patients had a total of 52 fractures on the mandible. In the 26 patients, mandibular fractures are related to impacted or unerupted teeth and remaning 15 patients also have mandibular fracture which is not related to any impacted or unerupted teeth.

The relationship between impacted or unerupted teeth and mandibular fracture status is summarized in Table 1. In group I, impacted or unerupted teeth contributed to $63.41 \%$ (26/41) of fractures. Patients having mandibular fractures and impacted or unerupted teeth had nearly a 1,73-fold increase the risk of mandibular fractures comparing with patients not having impacted or unerupted teeth. The impacted or unerupted teeth significantly increased the risk of fractures in Group I ( $\left.x^{2}=5,29, P=.0215\right)$.

Table 2 summarizes the degree of impactation as "complete" or "partial bony". In this study, fall caused, 18 (44\%); fight, 9 (22\%); motor vehicle accidents, $3(7 \%)$; sports, $3(7 \%)$; and others, $8(20 \%)$ of the mandibular fractures. Thus, it is seen that, was the most common cause of the mandibular fractures, with the effect of $44 \%$.

The incidence of mandibular fracture on impacted or unerupted teeth was evaluated. As it was seen, the most common fracture between the impacted or unerupted teeth was impacted third molars (54\%) (Table 3).

\section{DISCUSSION}

Consistent with other similar studies, the results of this study confirmed an increased risk of mandibular fracture when the impacted or un-

Table 2. The severity of tooth impaction.

\begin{tabular}{lccc}
\hline Variables & Group I & Group II & P \\
\hline Complete bony & 18 & 8 & \\
Partial bony & 8 & 7 & .49 \\
\hline
\end{tabular}

Table 3. Fracture amount associated with impacted or unerupted teeth.

\begin{tabular}{lc}
\hline Tooth Number & Fracture Amount \\
\hline 2 & 1 \\
3 & 6 \\
4 & 1 \\
5 & 3 \\
7 & 1 \\
8 & 14 \\
\hline
\end{tabular}


erupted teeth were present. It is hypothesized that the impacted or unerupted teeth increase the risk of mandibular fractures by occupying osseous space and thereby, the angle region is weakened. It is not true that the risk of mandibular fracture incidence depends on only one factor because it depends on the vector and also the amount of force, the musculature of the face, the architecture of the mandible and the presence or absence of M3. ${ }^{9}$

The hypothesis that M3 level of impaction further increases the risk of angle fractures originated with the work of Reitzik et al. ${ }^{8}$ The reasoning of this hypothesis is that when M3 occupies more osseous space, it weakens the mandible against the outside stresses. This compares the mandibular angle, when an impacted M3 is present, with a region of pathologic weakness similar to various conditions (i.e., presence of a tumour or cysts, periapical pathosis, hyperparathyroidism, Paget's disease, osteoporosis, and other metabolic conditions). ${ }^{10}$

Falls, motor vehicle accidents, fights, sports, and others cause to maxillofacial fractures commonly. In this study, falls were the most common cause of these fractures, comprising $44 \%$ of the etiology of the fractures. Similar to other investigators', ${ }^{11-13}$ we found that patient's age has an important role on the risk of fracture. Sixty-three of the patients were under the age of twenty years. In our study, only 10 women sustained fractures, whereas 31 men did.

Huelke et $\mathrm{al}^{14}$ reported that fractures occur more frequently in dentate than in edentulous regions of the mandible. Their findings were confirmed by Amaratunga's ${ }^{3}$ and Halazonetis's $s^{15}$ studies. Similar to these investigators, we found that the impacted or unerupted teeth in the dentate regions of mandible weakens the mandibular bone.

In this study, the most common mandibular fractures were seen as impacted or unerupted third molars teeth area. After that, the most common mandibular fracture was seen as impacted or unerupted canin teeth which have the longest root in the mandible.

\section{CONCLUSIONS}

The first specific aim of this study was to measure the association between the presence of impacted or unerupted teeth and the risk of mandibular fractures. We noted the significant association between the impacted or unerupted teeth presence and the risk of mandibular fractures statistically $(P=.0215)$. The results of this study confirmed that, if there are impacted or unerupted teeth, the risk of mandibular fractures will increase.

\section{REFERENCES}

1. Banks P. Killey's fractures of the mandible. 4th ed, London, Wright, 1991:1.

2. Rudderman RH, Mullen RL. Biomechanics of the facial skeleton. Clin Plast Surg 1992;19:11-29.

3. Amaratunga NAS. A comparative study of the clinical aspects of edentulous and dentulous mandibular fractures. $J$ Oral Maxillofac Surg 1988;46:3-6.

4. Safdar N, Meechan JG. Relationship between fractures of the mandibular angle and the presence and state of eruption of the lower third molar. Oral Surg Oral Med Oral Pathol 1995; 79:680-684.

5. Tevepaugh DB, Dodson TB. Are mandibular third molars a risk factor for angle fractures? A retrospective cohort study. J Oral Maxillofac Surg 1995;53:646-649.

6. Lee JT, Dodson TB. The effect of mandibular third molar presence and position on the risk of an angle fracture. $J$ Oral Maxillofac Surg 2000;58:394-398.

7. Peterson L, Ellis E, Hupp JR, Tucker MR. Contemporary Oral and Maxillofacial Surgery. The CV Mosby Company, St Louis, Washington, Toronto, 2003:223.

8. Reitzik M, Lownie JF, Cleaton-Jones P, Austin J. Experimental fractures of monkey mandibles. Int J Oral Surg 1978; 7:100-103.

9. Dodson TB. Cross-sectional area of the mandible: Discussion. J Oral Maxillofac Surg 1997;55:693-695.

10. Fuselier J, Ellis EE 3rd, Dodson TB. Do mandibular third molars alter the risk of angle fracture? J Oral Maxillofac Surg 2002;60:514-518.

11. Libersa P, Roze D, Cachart T, Libersa JC. Immediate and Late Mandibular Fractures after Third Molar Removal. Oral Maxillofac Surg 2002;60:163-165.

12. Perry PA, Goldberg MH. Late mandibular fracture after third molar surgery: A survey of Connecticut oral and maxillofacial surgeons. J Oral Maxillofac Surg 2000;58:858-861.

13. Krimmel M, Reinert $S$. Mandibular fracture after third molar removal. J Oral Maxillofac Surg 2000;58:1110-1112.

14. Huelke DF, Burdi AR, Eyman CE. Association between mandibular fractures and site of trauma, dentition, and age. J Oral Surg Anesth Hosp Dent Serv 1962;20:478-481.

15. Halazonetis JA. The 'weak' regions of the mandible. $B r J$ Oral Surg 1968;6:37-48. 